\author{
Thomas Brocker, \\ Annick Peter, \\ André Traunecker and \\ Klaus Karjalainen
} Basel Institute for Immunology+,
Basel

\section{New simplified molecular design for functional $T$ cell receptor}

\begin{abstract}
We have produced a chimeric single-chain $T$ cell receptor ( $T c R)$ that combines the specific antibody recognition function and $\mathrm{TcR} / \mathrm{CD} 3$ signaling properties within the same polypeptide chain. This hybrid molecule consisted of a single-chain antibody combining site that was connected over a short spacer to the transmembrane and cytoplasmic region of CD3\}. When expressed on $\mathrm{TcR}^{-}$or $\mathrm{TcR}^{+} \mathrm{T}$ cell hybridomas it could mediate recognition of relevent target cells and subsequent production of lymphokines; i.e. it could functionally replace the TcR/CD3 complex. Therefore, the single-chain TcR model presented here represents an interesting and useful means for the creation of $T$ cells with new specificities.
\end{abstract}

\section{Introduction}

The $T$ cell antigen receptor $(\mathrm{TcR})$ is a multiprotein complex composed of at least six different transmembrane proteins. The TcR $\alpha$ and $\beta$ chains are expressed as covalently linked $\alpha \beta$ heterodimers which provide the antigen specificity for $\mathrm{T}$ cells. While these proteins have large extracellular domains, their cytoplasmic portions contain only a few amino acids. The $\alpha$ and $\beta$ chains are noncovalently associated with the invariant subunits $\mathrm{CD} 3 \gamma,-\delta,-\varepsilon,-\zeta$ and/or $-\eta$ to form TcR/CD3 complexes [1]. These associated proteins have large intracellular domains and are thought to couple the process of antigen recognition to the signal transduction pathways of the T cell $[2,3]$. We wanted to bypass this complexity by creating a chimeric molecule that would retain both signaling and recognition functions within one polypeptide chain to generate an easily manipulatable system, which would allow us to change the specificity of a $\mathrm{T}$ cell at will.

Following several experiments which showed, that the intracellular portion of the $\mathrm{CD} 3 \zeta$ chain would be sufficient to induce activating signals after cross-linking of their artificial extracellular domains [4-6] we constructed a hybrid molecule, which unified the signaling properties of the $\mathrm{CD} 3 \zeta$ chain and the antigen-binding ability of an antibody. For this we used a single-chain (Fv) $\mathrm{mAb}$ form [7] derived from TR66, an $\mathrm{mAb}$ specific for human $\mathrm{CD} 3 \varepsilon$ [8], and grafted it onto a short polypeptide spacer (hinge) from the CD8 $\alpha$ chain. This chimeric extracellular domain was then finally fused to the transmembrane and cytoplasmic region of $\mathrm{CD} 3 \zeta$ to form the single-chain TCR "FvCD3 $\zeta$ ". When the construct coding for the single-chain TcR was transfected into $\mathrm{TcR}^{-}$or $\mathrm{TcR}^{+} \mathrm{T}$ cell hybridomas, the

[I 11540]

+ The Basel Institute for Immunology was founded and is supported by F. Hoffmann-La Roche Ltd. Basel, Switzerland

Correspondence: Thomas Brocker, Basel Institute for Immunology, Grenzacherstr. 487, CH-4005 Basel, Switzerland (Fax: 4161 6811304)

Abbreviation: Fv: Variable region combining site

Key words: $T$ cell receptor/Single-chain variable regions expressed chimeric molecule was able to mediate recognition of relevant target cells resulting in the production of IL- 2 and IL- 3 by the transfectants.

Since the antigen specificity of our model chimera can easily be changed by replacing its Fv domain by other Fv derived from any antibody, this system could be useful in creating $T$ cells of any desired specificity, bypassing the necessity of MHC restriction.

\section{Materials and methods}

\subsection{Construction of the chimeric FvCD3}

The vector pHBAPr-1-neo-FvCD3 $\zeta$ was designed to express the chimeric FvCD $3 \zeta$ construct under the control of the human $\beta$-actin promoter and bears a selectable neomycin marker. For its construction we removed the unique EcoRI restriction site in the plasmid pH $\beta \mathrm{APr}-1-n e o$ [9] by digesting it with EcoRI, blunting its cohesive ends and religating it. The polymerase chain reaction (PCR) was used to amplify a cDNA segment encoding for the cytoplasmic und transmembrane region of the murine $\mathrm{CD} 3 \zeta$ chain and 42 amino acids of the hinge region of murine $\operatorname{CD} 8 \alpha$ (oligonucleotide primers 5451, 5452). As a template for this reaction we used the plasmid CD8 $\zeta$ [6]. The primers of this reaction contained a SalI and a HindIII restriction site so that the amplified PCR product could be cloned after digestion with SalI and HindIII into the SalI-HindIII opened pHBAPr-1-neo(RI). The resulting plasmid was opened by SalI, blunted and recut with EcoRI to receive the EcoRI-digested PCR-amplified cDNA fragment coding for the VDJ heavy and VJ light chain region of the mAbTR66. As a template for this PCR amplification we used the plasmid FvCD3 $\mathrm{H} \gamma 3$ [10] and the oligonucleotide primers 5971 and 5979 with the latter containing an EcoRI cloning site.

The sequences of the used oligonucleotide primers are: 5'TCCTGTCGACAGTGGAATTCACTACTACCAAGCCAGTGCTGC3' (5451); 5'GCATAAGCTTGCCAGAAGACCCAAGAGCAGGG3' (5452); 5'TATGAGAATTCTTTCAGCTCCAGCTTGGTCCCAGC3' (5971); and 5 'ACTGCATGAGCTCGAGACAACACTGACTCTAACCATGGGA $3^{\prime}$ (5972). 


\subsection{Cell lines and monoclonal antibodies}

BW5147 $\alpha^{-} \beta^{-}$is a derivative of the AKR/J lymphoma BW5147 that fails to express the TcR $\alpha, \beta, \gamma, \delta$ and $\zeta / \eta$ genes $[6,11] .14 .3 \mathrm{~d}$ is a mouse $\mathrm{T}$ helper hybridoma (derived from the $T$ cell clone $V_{2-15}$ ) that recognizes a hemagglutinin peptide of influenza virus $A / P R / 8 / 34$ (H1N1) in the context of MHC class II I-E $E^{d}[12]$. The B lymphoma A20 [13] was used as antigen presenting cell for this peptide and Jurkat, a human acute T cell leukemia line (ATCC CRL 8162) and GC13, a human T cell clone (kindly provided by Dr. A. Lanzavecchia) were used as stimulators in the functional assays. All cells were cultured in IMDM containing $10 \%$ fetal calf serum.

For immunofluorescence and immunoprecipitations we used the following mAb: anti-FvCD3 (rat anti-FvCD3; Traunecker, unpublished data); 2C11 (hamster anti-mouse CD3e, [14]), F23.1 (anti-mouse TcR V $\beta 8$; [15]).

\subsection{Immunofluorescence}

Expression of cell surface proteins was assayed by indirect immunofluorescence. Cell suspensions of $1 \times 10^{5}$ viable cells were stained with $20 \mu \mathrm{g} / \mathrm{ml}$ of first-step mAb. After 30 min of incubation the cells were washed and incubated with the second-step FITC-conjugated goat anti-rat Ig (Southern Biotechnologies) at $10 \mu \mathrm{g} / \mathrm{ml}$. Stained cells were washed and fixed in $1 \%$ formaldehyde prior to cytofluorometric analysis using a FACScan (Becton Dickinson).

\subsection{Biochemical characterization of cell surface proteins}

Viable cells $\left(3 \times 10^{7}\right)$ were washed five times in washing buffer (PBS, $1 \mathrm{mM} \mathrm{MgCl}, 0.1 \mathrm{mM} \mathrm{CaCl}$ ) and then biotinylated for $30 \mathrm{~min}$ at $4^{\circ} \mathrm{C}$ in Sulfo-NHS biotin (Pierce, $0.5 \mathrm{mg} / \mathrm{ml}$ in washing buffer). After another five washes the biotinylated cells were lysed in lysis buffer containing $150 \mathrm{mM} \mathrm{NaCl}, 50 \mathrm{~mm}$ Tris, $1 \mathrm{mM}$ PMSF, $10 \mathrm{mM}$ iodoacetamide and $0.5 \%$ NP40 or $1 \%$ digitonin. The lysate was precleared overnight with protein G-Sepharose (Pharmacia) and the immunoprecipitation was performed for $2 \mathbf{h}$ with $10 \mu \mathrm{g}$ specific mAb followed by a $1-\mathrm{h}$ incubation with protein G-Sepharose. The precipitate was washed four times in lysis buffer and then boiled in $40 \mu \mathrm{l}$ of $1.5 \times$ Laemmli sample buffer prior to SDS-PAGE using $12.5 \%$ or gradient gels. The proteins were transferred from the SDS-PAGE to nitrocellulose which was then blocked in PBS, $0.4 \%$ Tween-20 (PBS-T) containing $5 \%$ nonfat dry milk. It was subsequently incubated for $1 \mathrm{~h}$ in PBS-T containing $0.08 \mu \mathrm{g} / \mathrm{ml}$ streptavidin-horseradish peroxidase conjugate (Southern Biotechnology). The blot was washed three times for $5 \mathrm{~min}$ in PBS-T before addition of the ECL chemoluminescence reagent (Amersham) and the nitrocellulose was exposed to an X-ray film (Kodak) for $30 \mathrm{~s}$ to $3 \mathrm{~min}$.

\subsection{Stimulation of $T$ cell transfectants}

In each assay $5 \times 10^{4}$ responder cells were stimulated with different amounts of human $T$ cells, which were fixed before the experiment by a $30-\mathrm{s}$ treatment with $0.05 \%$ glutaral- dehyde. When the APC A20 was used to present influenza virus hemagglutinin peptide, the A20 cells were irradiated $(8000 \mathrm{rad})$ before being used at $5 \times 10^{4}$ per assay with various amounts of peptide.

In each instance the supernatants of the stimulation assays were collected $24 \mathrm{~h}$ after the start of the experiment and tested for their level of IL-2 and IL-3 [16, 17] on the IL-2-dependent cell line CTL.L [18] and the IL-3-dependent cell line DA-1 [19].

\section{Results and discussion}

\subsection{General strategy}

To investigate if the TCR/CD3 multiprotein complex could be replaced functionally by a single-chain chimeric polypeptide, we designed a chimeric gene, which could direct the synthesis of a protein consisting of the cytoplasmic and transmembrane region of mouse $C D 3 \zeta$, a 42-amino acid segment of mouse $C D 8 \alpha$ as extracellular hinge region and the antigen combining site of the mAb TR66 (Fig. 1B); TR66 is specific for the human CD $3 \varepsilon$ chain [8], which was already successfully modified to bispecific single-chain $\mathrm{Fv}$ molecules by Traunecker et al. [10]. The final construction (Fig. 1A) was then transfected by protoplast fusion [20] into the $\mathrm{T}$ hybridomas $\mathrm{BW \alpha}^{-} \mathrm{\beta}^{-}\left(\mathrm{TcR}{ }^{-}\right)$and $14.3 \mathrm{~d}\left(\mathrm{TcR}^{+}\right)$ and G418-resistant stable transfectants were isolated and analyzed for expression and function of the chimeric single-chain $\mathrm{TcR}$.

\subsection{Surface expression and protein characterization}

The immunofluorescence analysis (Fig. 2) shows that the parental hybridomas $\mathrm{BW}^{-} \boldsymbol{\beta}^{-}$(Fig. 2A) and 14.3d (Fig. 2C) did not stain with the mAb anti-FvCD3, while the

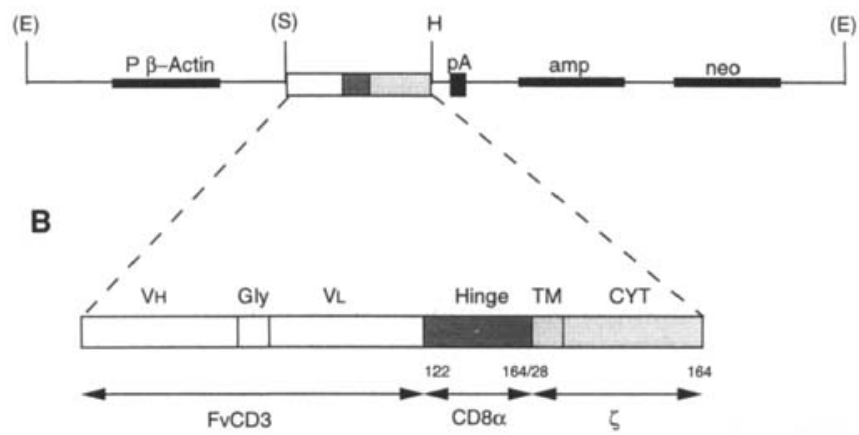

Figure 1. (A) A schematic representation of the chimeric FvCD3ל construct. The FvCD3 $\zeta$ cDNA is expressed under the control of the human $\beta$-actin promoter (P $\beta$-Actin). The plasmid further carries selectable neomycin and ampicillin resistance genes (neo, amp) and a polyadenylation site (pA) at the $3^{\prime}$ end of the cDNA. The indicated restriction sites are EcoRI (E), SalI (S) and HindIII (H). Restriction sites in parentheses have been removed by blunt end cloning. (B) The predicted structure of the FvCD3 $\zeta$ polypeptide. The FvCD3 portion consists of the heavy and light chain variable regions ( $\mathrm{VH}, \mathrm{VL}_{\mathrm{L}}$ ) of TR66 connected by a glycine linker (Gly) [10]. This Fv domain is followed by amino acid residues 122 to 164 of the hinge region of $C D 8 \alpha$ (numbering according to [22]) and the transmembrane (TM) and cytoplasmic portion (CY) of the $\zeta$ chain from residue 28 to 164 (numbering according to [23]). 

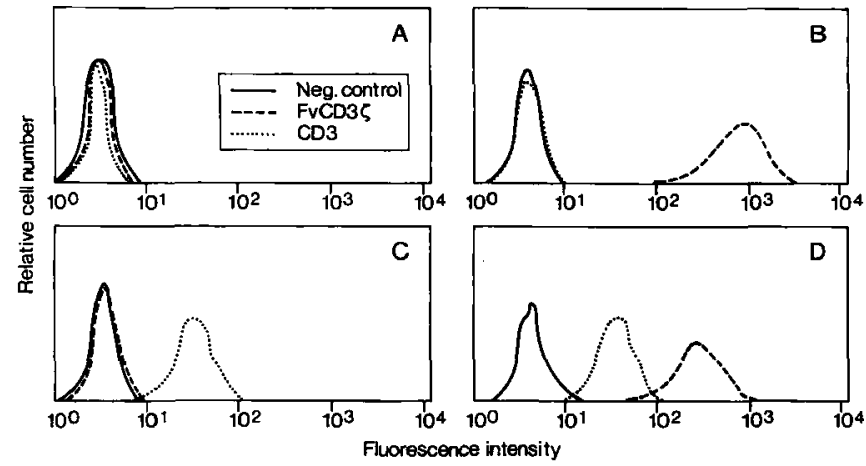

Figure 2. Flow cytometry of cells stained with antibodies specific for mouse $\mathrm{CD} 3$ (mAb 2C11, dotted lines), FvCD3 $\zeta$ (dashed lines) and second-step fluorescein-conjugated antibody only FITC-goat anti-rat, solid lines). (A) shows the untransfected parental hybridoma $B W \alpha^{-} \beta^{-}$, while (B) shows the staining of the $B W \alpha^{-} \beta^{-}$ transfectant BW TF1. The cytofluometric analysis of the $\mathrm{TcR}^{+}$ hybridoma $14.3 \mathrm{~d}$ and its transfectant $14.3 \mathrm{~d}$ TF1 are shown in (C) and (D), respectively.

transfectants of each hybridoma (Fig. 2B,C) showed a surface staining indicating that the FvCD3 determinant was expressed on their surface. In contrast to $14.3 \mathrm{~d}$ and its transfectants (Fig. 2C,D), the $\mathrm{BW} \alpha^{-} \beta^{-}$transfectants (Fig. 2B) as well as the parental BW $\alpha^{-} \beta^{-}$(Fig. 2A) did not express endogenous TcR/CD3 complexes, as monitored with the mouse CD3e-specific mAb $2 \mathrm{C} 11$ [14].

Theoretically, several possible forms of chimeric singlechain TcR could be expressed on the surface of the transfectants: while the $\mathrm{BWa}^{-} \boldsymbol{\beta}^{-}$transfectants should express FvCD3 $\zeta$ independently of endogenous TcR/CD3 complexes, an association could appear in the 14.3 transfectants. FvCD3 $\zeta$ could then associate with endogenous TcR/CD3 complexes on the cell surface by charge pairing of its $\mathrm{CD} 3 \xi$ transmembrane region [21]. Furthermore, this $\mathrm{CD} 3 \zeta$ transmembrane region could potentially permit covalent disulfide linkage between the single-chain FvCD3 $\zeta$ and endogenous wild-type $\mathrm{CD} 3 \xi$ chains, resulting in the formation of $\mathrm{CD} 3 \zeta$-FvCD $3 \xi$ heterodimers. To investigate which of these was expressed on the surface of our transfectants, we biotinylated the cell surface of these cells, lysed them either in $0.5 \%$ NP40 or $1 \%$ digitonin and used the lysates for immunoprecipitations.

The SDS-PAGE analysis of the immunoprecipitations under reducing conditions, followed by a Western blot analysis (Fig. 3, lanes 1,2), showed that the FvCD3 protein had an apparent molecular weight of 60000 on the BW transfectant when the FvCD3-specific mAb was used for precipitation. When analyzed under nonreducing conditions the FvCD $3 \xi$ protein seemed to have a dimeric and to a lesser degree tetrameric form on the $B W \alpha^{-} \beta$ transfectant (Fig. 3, lane 4).

Surprisingly we could only detect homodimers on the surface of the 14.3d transfectant (Fig. 3, lane 7) and no $\mathrm{CD} 3 \zeta$-FvCD $3 \zeta$ heterodimers; these findings suggest that those kinds of heterodimers are either not transported to the cell surface or they are not formed at all.

To detect the eventual associations between FvCD $3 \zeta$ and endogenous TcR/CD3 components we lysed the biotin surface-labeled $14.3 \mathrm{~d}$ transfectant with $1 \%$ digitonincontaining lysis buffer, which preserves the TcR/CD3 association. The digitonin lysate of the 14.3 transfectant was either used for precipitation with the anti FvCD3-mAb (Fig. 3, lanes 5, 6) or with F23.1 [15], and antibody specific for the V $\beta 8$ segment of the TcR $\beta$ chain (Fig. 3, lanes 7, 8). The SDS-PAGE-analysis under nonreducing conditions revealed, that the F23.1 precipitation pattern of the untransfected $14.3 \mathrm{~d}$ and its transfectant were identical: the major protein was the TcR $\alpha \beta$ heterodimer while an additional coprecipitated $\mathrm{FvCD} 3 \xi$ homodimer on the transfectant could not be detected (Fig. 3, lanes 5,6). Vice versa the immunoprecipitation with anti-FvCD3 did not coprecipitate TcR polypeptides from the $14.3 \mathrm{~d}$-transfectant (Fig. 3, lane 7).

This analysis of the $14.3 \mathrm{~d}$ transfectant indicated, that the chimeric FvCD3 $\zeta$ protein was expressed independently from endogenous $T c R / C D 3$ proteins and that its major form was a homodimer. Therefore, the presence of the endogeneous TcR/CD3 complexes of the 14.3d transfectants should not sterically hinder the function of the FvCD3 3 polypeptide and vice versa.

\subsection{Functional tests of the FvCD3 $\zeta$ chimera}

According to the experiments of Irving and Weiss [4] and Romeo and Seed [5] the presence of the intracellular portion of $\mathrm{CD} 3 \zeta$ was sufficient to activate their transfectants when the extracellular domains of their chimeric proteins $\mathrm{CD} 8 \zeta$ or CD4 $\zeta$ were cross-linked by either CD8specific $\mathrm{mAb}$ or gp120. We wanted to extend these findings and test if our transfectants would be able to recognize antigen (human CD3 $\varepsilon$ ) through their FvCD3 domain and if this was sufficient to activate the $\mathrm{T}$ cell transfectants.

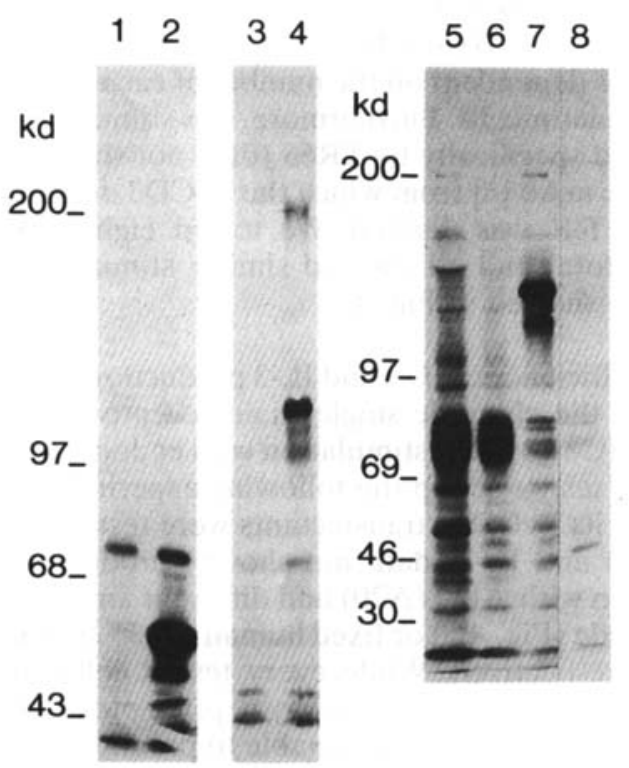

Figure 3. Biochemical analysis of cell surface proteins: $B W \alpha^{-} \beta^{-}$ (lanes 1,3) and one of its transfectants BWTF1 (lanes 2,4) as well as 14.3d (lanes 6,8) and its transfectant 14.3dTF1 (lanes 5, 7) were cell surface biotinylated, lysed in either $0.5 \%$ NP40- (lanes $1-4$ ) or $1 \%$ digitonin- (lanes 5-8) containing lysis buffer. The lysates were used for immunoprecipitations with either FvCD3-specific mAb (lanes 1-4, 7, 8) or TCR-specific mAb F23.1 (lanes 5, 6). 

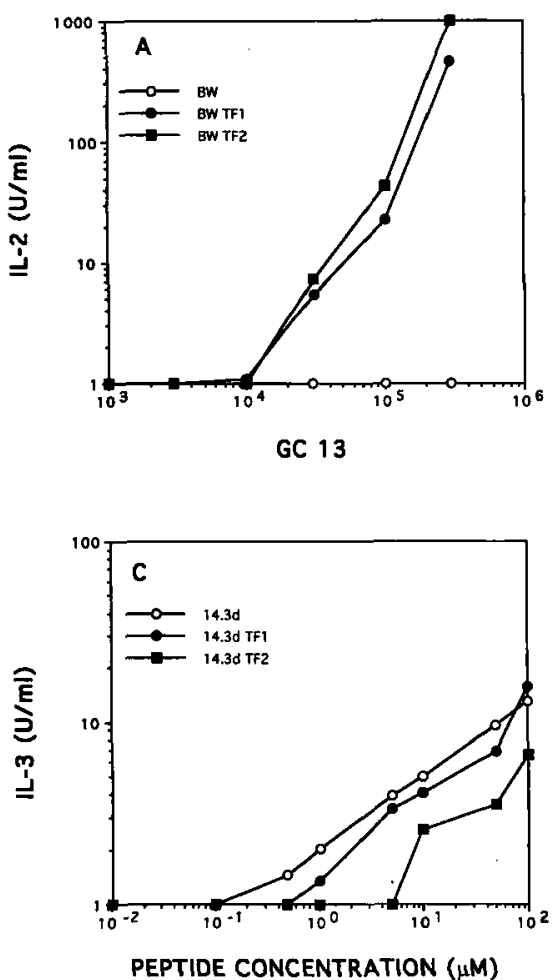

PEPTIDE CONCENTRATION $(\mu M)$
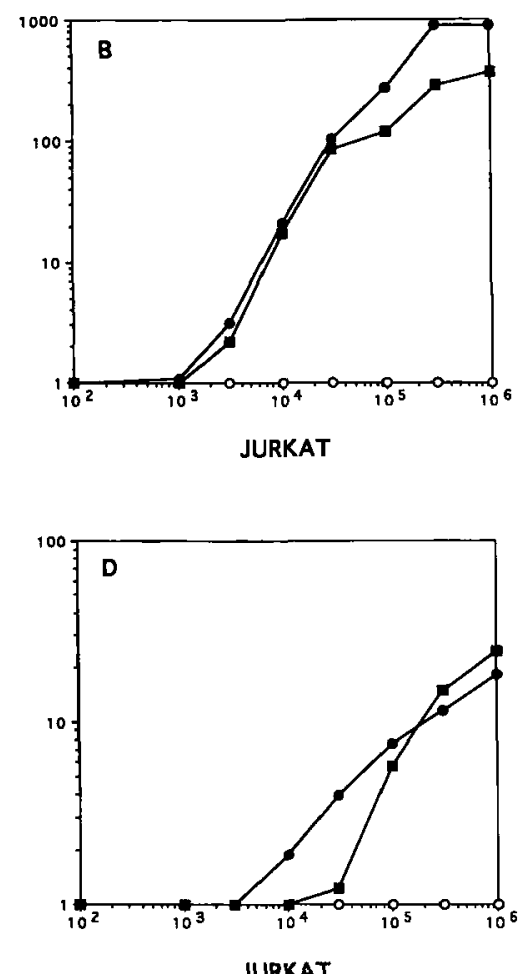

Figure 4. IL-2 (A, B) and IL-3 (C, D) production of $\mathrm{BW}$ and $14.3 \mathrm{~d}$ cells transfected with the chimeric FvCD3 3 gene constructs after stimulation with the fixed human $T$ cells GC 13 (A), Jurkat (B, D) or peptide antigen presented by $\mathrm{A} 20$ (C). The parental cell $B W \alpha^{-} \beta^{-}$(open circles) and two transfected subclones BW TF1 (closed circles) and BW TF2 (closed squares) were incubated with the indicated numbers of GC 13 (A) and Jurkat (B); 14.3d (open circles) and two of its transfectants [14.3d TF1 (closed circles), 14.3d TF2 (closed squares)] were stimulated either by antigen-presenting cell and different peptide concentrations (C) or the indicated numbers of fixed Jurkat cells (D). After $22 \mathrm{~h}$ the supernatants were assayed for their content of IL-2 or IL-3 using IL-2-dependent CTL.L or IL-3-dependent DA- 1 cells.
As stimulators we used Jurkat (Fig. 4B, D), a human CD3 ${ }^{+}$ T cell lymphoma and GC13 (Fig. 4A), a human $T$ cell clone (A. Lanzavecchia, unpublished data). Both stimulators were fixed before the functional assays in order to make sure, that they could not be a source of lymphokine production (data not shown). Fig. $4 \mathrm{~A}$ and $\mathrm{B}$ shows the results of the stimulation of $B W \alpha^{-} \beta^{-}$and two of the $B W$ transfectants. While the untransfected parental hybridoma was apparently neither activated by the stimulator cells GC13 (Fig. 4A), nor Jurkat (Fig. 4B), the two transfectants produced IL-2 (Fig. 4A, B) and IL-3 (data not shown) in a manner which was dependent on the number of each of the two types of stimulator cells. Furthermore, this stimulation could be inhibited specifically by TR66 (data not shown), the CD3e-specific mAb [8] from which the FvCD3 domain of our chimeric TcR was derived. We tested eight BW transfectants in total and all showed similar stimulation patterns to those showed in Fig. 4.

To compare the efficiency of IL-2 and IL-3 production after stimulation over the chimeric single-chain receptor with conventional MHC-restricted stimulation with endogenous TcR/CD3 complexes we set up the following experiments: 14.3d and two of its FvCD3 $\zeta$ transfectants were tested for IL-3 (Fig. 4C, D) and IL-2 (data not shown) production during stimulation with APC (A20) and different amounts of antigenic peptide (Fig. $4 \mathrm{C}$ ) or fixed human $\mathrm{CD}^{+}$Jurkat cells (Fig. 4D), respectively. While every tested cell was stimulated in a similarly efficient way by peptide plus A20 (Fig. 4C), only the transfectants were able to produce IL-3 (Fig. 4D) and IL-2 (data not shown) during stimulation by fixed Jurkat cells. Both kinds of assay showed that the production of lymphokines was either dependent on peptide concentration or on the number of stimulator cells, and indicated that the additional anti-human CD3 specificity on the 14.3d transfectants is caused by the expression of the single-chain TcR. A comparison of the stimulation efficien- cies through endogeneous TcR (Fig. 4C) and FvCD3 $\xi$ single-chain TcR (Fig. 4D) shows that both signaling machineries seem to be similarly efficient in inducing lymphokine production in the same cell. While the peptide antigen presented by $\mathrm{A} 20$ is recognized in an MHC-restricted way, the CD3 recognition through FvCD3 $\zeta$ resembles an antibody-antigen interaction where no restricting $\mathrm{MHC}$ is needed.

\section{Concluding remarks}

Here we have demonstrated that the biological activities of helper $\mathrm{T}$ cell hybridomas can be directed by a single polypeptide chain that is able to recognize antigen and to transduce activating signals into the cell, which result in the production of biologically active lymphokines, in manners indistinguishable from normal antigen-specific stimulation.

The anti-human CD3 specificity used in our experiments can be easily exchanged with Fv, of other desired specificities derived from any existing $\mathrm{mAb}$. Therefore, the singlechain TcR model presented here represents an interesting and useful means for the creation of $T$ cells with every desired specificity at will.

While our manuscript was under preparation Eshhar et al. published a similar study [24] with comparable results. Their findings and those of Moritz et al. (personal communication) combined with ours suggest that the single-chain TcR technology is generally applicable.

We would like to thank Drs. Peter Lane and Antonio Lanzavecchia for critical reading of the manuscript.

Received February 25, 1993; in revised form March 22, 1993. 


\section{References}

1 Clevers, H., Alarcon, B., Willeman, T. and Terhorst, C., Annu. Rev. Immunol. 1988. 6: 629.

2 Weissman, A. M., Frank, S. J., Orloff, D. G., Mercep, M., Ashwell, J. D. and Klausner, R. D., EMBO J. 1989. 8: 3651.

3 Frank, S. J., Niklinska, B. B., Orloff, D. G., Mercep, M., Ashwell, J. D. and Klausner, R. D., Science 1990. 249: 174.

4 Irving, B. A. and Weiss, A., Cell 1991. 64: 891.

5 Romeo, C. and Seed, B., Cell 1991. 64: 1037.

6 Wegener, A.-M. K., Letourneur, F., Hoeveler, A., Brocker, T., Luton, F. and Malissen, B., Cell 1992. 68: 83.

7 Huston, J. S., Levinson, D., Mudgett-Hunter, M., Tai, M.-S., Novotny, J. Margolies, M. N., Ridge, R. J., Bruccoleri, R. E., Haber, E., Crea, R. and Oppermann, H., Proc. Natl. Acad. Sci. USA 1988. 85: 5897.

8 Lanzavecchia, A. and Scheidegger, D., Eur. J. Immunol. 1987. 17: 105.

9 Gunning, P., Leavitt, J., Muscat, G., Sy, N. G. and Kedes, L., Proc. Natl. Acad. Sci. USA 1987. 84: 4831.

10 Traunecker, A., Lanzavecchia, A. and Karjalainen, K., EMBO J. 1991. 10:3655.

11 White, J., Blackman, M., Bill, J., Kappler, J., Marrack, P., Gold, D. and Born, W., J. Immunol. 1989. 143: 1822.
12 Hackett, C. J., Dietzscholt, B., Gerhard, W., Ghrist, B., Knorr, R., Gillessen, D. and Melchers, F., J. Exp. Med. 1983. 158: 294.

13 Walker, E. N., Warner, N. L., Chestnut, R., Kappler, J. and Marrack, P., J. Immunol. 1982. 128: 2164.

14 Leo, O., Foo, M., Sachs, D. H., Samelson, L. E. and Bluestone, J. A., Proc. Natl. Acad. Sci. USA 1987. 84: 1374.

15 Sterz, U., Rammensee, H. G., Benedetto, J. and Bevan, M., J. Immunol. 1985. 134: 3994.

16 Mosmann, T., J. Immunol. Methods 1983. 65: 55.

17 Tada, H., Shiho, O., Kuroshima, K.-L., Koyama, M. and Tsukamoto, K., J. Immunol. Methods 1986. 93: 157.

18 Gillis, S., Ferm, M. M., Ou, W. and Smith, K., J. Immunol. 1978. 120: 2027.

19 Ihle, J. N., Rein, A. and Mural, R., in Klein, G. (Ed), Advances in viral Oncology, Raven Press, New York 1984, p. 95.

20 Oi, V. T., Morrison, S. L., Herzenberg, L. A. and Berg, P., Proc. Natl. Acad. Sci. USA 1983. 80: 825.

21 Cosson, P., Lankford, S. P., Bonifacino, J. S. and Klausner, R. D., Nature 1991. 351: 414.

22 Zamoyska, R., Vollmer, A. C., Sizer, K. C., Liaw, C. W. and Parnes, J. R., Cell 1985. 43: 153.

23 Weissman, A. M., Baniyash, M., Hou, D., Samelson, L. E., Burgess, W. H. and Klausner, R. D., Science 1988. 239: 1018.

24 Eshhar, Z., Waks, T., Gross, G. and Schindler, D. G., Proc. Natl. Acad. Sci. USA 1993. 90: 720. 